\title{
CALIDAD EN LOS SERVICIOS SOCIALES DE ACOGIMIENTO FAMILIAR
}

\author{
The quality of specialized family placement social services \\ M. ${ }^{\text {a }}$ Victoria OCHANDO RAMÍREZ ${ }^{1}$, AnNa Rucabado SAla ${ }^{2}$, \\ ANA RAQUEl ORTEGA MARTÍNEZ ${ }^{3}$
}

\section{Resumen}

Introducción: El objetivo de esta investigación ha sido determinar atributos de calidad propios para Servicios Sociales Especializados a través de la perspectiva de los profesionales.

Material y métodos: Se ha realizado una evaluación de calidad en un servicio de acogimiento familiar. Primeramente, se ha hecho un estudio de las expectativas y percepciones mediante las dimensiones de calidad de la herramienta SERVQUAL. Además en el marco del Modelo EFQM en su adaptación al sector público y las entidades de voluntariado de la Junta de Andalucía, se ha desarrollado un estudio exploratorio de la entidad y una autoevaluación de calidad.

Resultados: Los datos muestran la importancia de la evaluación de la calidad en estos servicios y las ventajas de combinar distintas técnicas para obtener información. De las respuestas de los participantes se han extraído una serie de atributos de calidad que generan calidad al servicio y a los profesionales.

Discusión: La evaluación ha permitido identificar tres puntos clave en la calidad del servicio de acogimiento familiar que son comunes para el resto de Servicios Sociales Especializados. Al mismo tiempo, se han identificado las dificultades que presentan los modelos de calidad para aplicarse directamente a estos servicios. Los resultados obtenidos avalan la necesidad de adaptar los modelos de calidad de referencia a cada realidad introduciendo indicadores de calidad específicos.

Palabras clave: calidad, evaluación, atributos de calidad, acogimiento familiar, Servicios Sociales Especializados.

\begin{abstract}
Introduction: The main goal of this study is to obtain specific quality dimensions for specialized social services from a professional perspective.

Material and Methods: In this study, quality assessment was conducted in the area of family placement services. The first step was a study of the expectations and perspectives using the service dimensions of the SERVQUAL tool. In addition, using the EFQM Excellence Model in an adaptation created for the public sector and volunteer organizations of the Junta of Andalusia, an exploratory study was carried out about the functioning of the organization and a quality self-evaluation.

Results: The data shows that quality assessment is very important for these services. The different means of approximating quality provides advantages. Participants have contributed to obtain quality dimensions for quality service and for professional satisfaction.

Discussion: this research study reveals three key aspects for quality in family placement services, which reveals the great similarity between specialized social services. There is also a difficulty in understanding and applying the existing quality models in these services. It suggests that quality models need to be adapted by introducing some specific indicators in order to be more effective.
\end{abstract}

Keywords: Quality, Quality dimensions, Family Placement, Specialized Social Services.

Recibido: 20/07/2015

Aceptado: 18/09/2015

Publicado: 03/12/2015

1. Universidad de Jaén. Departamento de Psicología. Jaén, España, Campus Las Lagunillas S/N, 23071 Jaén, España, 9532121 21, E-mail: mvochando@gmail.com

2. Universidad de Jaén. Departamento de Psicología. Jaén, España.

3. Universidad de Jaén. Departamento de Psicología. Jaén, España. 


\section{Introducción}

Hoy en día es muy frecuente recurrir a la calidad para apreciar el buen hacer de una organización o valorar un determinado servicio. Del mismo modo, cada vez más esta filosofía va impregnado los discursos de todos los ámbitos. Sin embargo, cuando nos plantemos la cuestión de la calidad en Servicios Sociales, por lo general, surge un vacío. Esta incertidumbre se debe en gran medida a las escasas iniciativas de gestión de la calidad que se han desarrollado en este contexto (Medina-Tornero, 2006; Parlamento Europeo, 2011), a diferencia de lo que sucede con otros Sistemas de Protección Social como puede ser Salud o Educación. Del mismo modo, tampoco se han estudiado cuáles han de ser los atributos de calidad que han de darse en este sistema.

En el caso de Servicios Sociales Especializados la cuestión responde al mismo patrón, es decir, la gestión de la calidad no se encuentra generalizada. Esto nos ha llevado a plantearnos el objetivo de determinar en los atributos de calidad de Servicios Sociales Especializados propios desde el punto de vista de los profesionales.

Además en este segundo nivel de atención, nos encontramos con otras limitaciones que tienen una gran repercusión en la calidad de los servicios prestados, y en consecuencia, en la calidad de vida de los ciudadanos que necesitan de ese servicio y en la calidad laboral de quienes lo prestan (Barranco, 2011).

En primer lugar, el hecho de que estos servicios sean tan heterogéneos entre sí ha impedido que se desarrollen unos criterios mínimos de calidad comunes para todos ellos. La heterogeneidad responde a dos circunstancias: una, referente a las múltiples formas de gestión y otra, a la titularidad de las entidades prestadoras que van desde los público hasta las empresas de la iniciativa privada y las entidades de voluntariado. Además, hay que mencionar la gran variedad de necesidades a las que hacer frente y que por lo general, se les da respuesta a través de la intervención especializada que se presta a sectores de población como las personas sin hogar, drogodependientes, tercera edad, discapacidad, menores, minorías, etc. Esta amalgama de factores ha ocasionado que la implantación de la calidad haya seguido patrones diferentes (Fundación Edad y Vida, 2008), basados fundamentalmente en la aplicación de modelos como las Normas ISO de la Organización Internacional de Estandarización, el modelo EFQM de la Fundación Europea de la Gestión de la Calidad, y la Norma ONG con calidad, diseñada por INTRESS y actualmente gestionada por ICONG. La gestión de la calidad ha predominado fundamentalmente en las entidades de gran tamaño y en especial, dentro de los sectores de personas mayores y con discapacidad. Además estas entidades 
ante la situación actual, han visto un incremento de la demandas (European Commission, 2013) que no se ha visto correspondida en todas las ocasiones por las prioridades políticas.

Pese a ello, las iniciativas de calidad han tenido en muchas ocasiones como objetivo en sí mismo la certificación de calidad. Esto tiene que ver con la situación generalizada que atraviesan los Servicios Sociales Especializados, ya que no tienen establecidas las vías de financiación como sucede con los Servicios Sociales Comunitarios. De ahí que para mantener determinados servicios sea necesario recurrir a la búsqueda de recursos, entre los cuales se encuentran las subvenciones públicas. Esto ocasiona que las entidades opten por certificar la calidad de sus servicios para hacer frente a la competencia del resto de entidades (Miranda, Chamorro y Rubio, 2007).

Relacionado con lo anterior, las entidades se encuentran además con las dificultades que entraña por sí mismo la implementación de sistemas de calidad: el lenguaje resulta ajeno a los Servicios Sociales Especializados, la aplicación de los modelos de calidad es compleja y también existen prejuicios relacionados con la normalización y sus ventajas entre las organizaciones y entre los propios profesionales del sector. Es decir, los modelos de referencia de calidad, genéricos para todo tipo de servicios no han logrado integrar las singularidades de estos servicios, donde el componente interrelacional adquiere una gran significación.

Precisamente por este motivo, se ha visto oportuno conocer hasta qué punto los modelos de calidad eran aplicables al contexto de Servicios Sociales Especializados; o si por el contrario, era necesario incorporar a dichos modelos otros indicadores de calidad propios. Igualmente se quería comprobar si los indicadores debían ser exclusivos para el acogimiento familiar o si podrían ser compartidos por los Servicios Sociales Especializados. Para ello, se ha realizado una evaluación de calidad que se ha centrado en una entidad de Servicios Sociales Especializados de pequeño tamaño y donde las experiencias de calidad han tenido poca repercusión debido a la privacidad y confidencialidad que exigen las situaciones que abordan. En concreto, se ha optado por una Institución Colaboradora de Integración Familiar (I.C.I.F.) responsable de gestionar los acogimientos familiares en una provincia andaluza.

El acogimiento familiar es una medida de protección que da respuesta a aquellas situaciones en que niños y niñas no pueden o no deben estar con sus padres biológicos y pasan a vivir temporal o permanentemente por otro núcleo familiar diferente al suyo de origen, que se encarga de su cuidado y educación. Se trata de evitar que el menor que se encuentre en una situación de desprotección, ingrese o permanezca en un Centro de Menores y con ello 
evitar así su institucionalización. El acogimiento familiar se produce durante el tiempo que se estudia la recuperabilidad de la familia de origen a través de la familia extensa del menor o de una familia ajena.

En estos servicios, la calidad supone no sólo la oportunidad de oír la voz y las necesidades de los usuarios, tal y como apunta Arenas (2015) en su estudio de calidad percibida, sino que supone ir mucho más allá. La gestión de la calidad en Servicios Especializados como el que nos ocupa, implica favorecer el bienestar de los menores por encima de todo y establecer procesos de calidad donde los plazos son más importantes si cabe, ya que se trata de minimizar los efectos no deseados de la protección (Cruz Roja Española, 2010) a estos menores que atraviesan circunstancias muy complejas (Del Valle, Bravo y López, 2009) derivadas de la separación familiar, el intervencionismo profesional y los múltiples cambios en las esferas de su vida. Además la protección de los menores ha de estar por encima de cuestiones como la disponibilidad presupuestaria, por lo que también es importante evitar incurrir en gastos innecesarios derivados de duplicidades y errores producidos por la no calidad.

Partiendo de estas premisas, se ha realizado una evaluación de calidad en la Asociación de Acogimiento Familiar. Esta entidad, se compone de un presidente y una coordinadora, y un equipo compuesto por cinco trabajadores, todos ellos con profesiones técnicas de carácter psicológico, social y legal. Además, el hecho de que la entidad evaluada dependa de la Administración Pública, a través de la Consejería de Igualdad, Salud y Políticas Sociales de la Junta de Andalucía, implica la asunción de los principios de calidad que se promueven para los servicios públicos. Esto es, situar al ciudadano en el centro de las actuaciones, promover la mejora continua, aprender de la experiencia propia o ajena, simplificar procesos y realizar evaluaciones de calidad. En este sentido, la evaluación realizada se ha centrado en la aplicación de los conceptos de la calidad y se hecho desde perspectiva de uno de los modelos de calidad, tal y como se explicará más adelante.

Esta evaluación no ha estado exenta de dificultades, ya que el acceso a la información en los casos de menores en situación de desamparo implica un elevado grado de confidencialidad, dada la sensibilidad del tema. Asimismo el lograr la apertura de la organización y de los profesionales para evidenciar lo que se está haciendo bien y aquello susceptible de ser mejorado, representa una dificultad añadida; al igual que el cerciorarse de que los indicadores de calidad que se propongan como específicos para el servicio de acogimiento, sean los que tienen que ser. 


\section{Material y métodos}

El proceso de evaluación de calidad se ha realizado desde la perspectiva de los profesionales de la entidad, con el fin de indagar en sus expectativas y percepciones del servicio. Para ello, se ha empleado la metodología cualitativa, por ser la más recomendada para este tipo de evaluaciones (Ministerio de Administraciones Públicas, 2006).

En esta ocasión, se ha querido realizar una evaluación de calidad que abordase distintos aspectos para obtener una visión holística del acogimiento familiar. Se ha visto que las evaluaciones de calidad centradas exclusivamente en el estudio de la satisfacción de alguno de los grupos de interés realizadas en algunos Servicios Sociales Especializados, no aportan datos sobre los aspectos de mejora de manera concreta.

En especial, estas limitaciones se presentan con el uso de la metodología cuantitativa. Por ejemplo, es habitual emplear la herramienta SERVQUAL desarrollada por Zeithman, Parasunaman y Berry (1992). Esta herramienta, es un cuestionario de 22 ítems que mide a través de una escala tipo Likert del 0 al 10 que permite evaluar la satisfacción con el servicio a través de cinco dimensiones: elementos tangibles, fiabilidad, capacidad de respuesta, seguridad y empatía. Sin embargo, aunque esta herramienta posibilita la obtención de una puntuación numérica y proporciona información sobre la satisfacción del servicio a partir de la diferencia existente entre las expectativas y percepciones, desde nuestro punto de vista, los datos que proporciona la herramienta sobre dichas dimensiones son más bien escasos y estructurados, no dejando cabida para otros atributos de calidad.

Puesto que con la evaluación realizada se quería conocer si existían otros indicadores de calidad que pudieran ser útiles para el servicio evaluado, se optó por utilizar las dimensiones que propone el modelo SERVQUAL para indagar sobre las expectativas y percepciones que tienen los profesionales sobre cada una de ellas. Para ello, se utilizado como técnica el grupo de discusión. Esta técnica ha favorecido que los participantes aportasen información sobre la satisfacción con las distintas dimensiones y los elementos que la engloban. Al mismo tiempo se han proporcionado detalles concretos sobre el servicio a partir de las dimensiones y sobre otros atributos de calidad relevantes. Se han realizado dos grupos, que responden a la totalidad de la población investigada: uno mixto compuesto por los dos directivos y los profesionales de la entidad, y otro exclusivo para el equipo de los profesionales de la entidad con objeto de contrastar la información. Las sesiones de discusión han durado hora y media cada una, y ha predominado el criterio de saturación de la información. 
De manera complementaria, se han utilizado otras técnicas para la evaluación de la calidad que han posibilitado conocer la calidad del servicio a nivel interno y en relación al contexto en el que se desenvuelve: el Servicio de Protección de Menores y otros sistemas asociados como son Salud, Educación y Servicios Sociales Comunitarios o de atención primaria.

De forma exploratoria, se ha indagado en el funcionamiento de la entidad a través de sus procesos. Se han utilizado técnicas como la observación, la revisión documental y las entrevistas semiestructuradas a todos los profesionales de la entidad en distintos momentos. Además, teniendo en cuenta que la normativa de calidad exige la utilización de un modelo de referencia para la evaluación de calidad, se ha empleado como guía el Modelo Europeo de Excelencia (EFQM) en su adaptación para el sector público y las organizaciones de voluntariado de la Junta de Andalucía (Dirección General de Administración Electrónica y Calidad de los Servicios, 2004b).

Posteriormente, se ha realizado una Autoevaluación de Calidad mediante el cuestionario-formulario establecido para los servicios públicos de la Junta de Andalucía (Dirección General de Administración Electrónica y Calidad de los Servicios, 2004a) que responde a los criterios agentes y resultados del modelo de calidad citado, que se ha rellenado de forma individual por parte de todos los participantes, con la ayuda inicial de la investigadora responsable. Después se ha realizado una reunión de consenso con los líderes.

\section{Resultados}

Los participantes consideran que la calidad aporta ventajas para el acogimiento que podría facilitarles su trabajo diario: «Puede permitirnos mejorar el servicio a la gente de fuera y de dentro»; «Puede hacer que todo sea más dinámico, más fácil y más seguro»; «Con la calidad sabes cómo puedes responder a cada situación»; «Mejora el funcionamiento a todos los niveles». El deseo de mejorar es un elemento que se reitera a lo largo de las distintas fases de la investigación, al igual que los aspectos que consideran que aportan calidad al servicio de acogimiento familiar.

El estudio de las expectativas y percepciones de los profesionales evaluados a partir de las dimensiones de SERVQUAL ha mostrado una gran satisfacción en la mayoría de las dimensiones, otorgando mucho interés a los aspectos relacionados con la atención profesional y el compañerismo: «Interés por el caso y que te preocupas»; «Buen ambiente, cooperación, poder contar con el otro, profesionalidad»; «Hay que destacar el compañerismo que tenemos y el apoyo». También ponen mucho interés en la mejora de los aspectos tangibles de la entidad: "Convendría separar las visitas de los despachos, se 
escucha el ruido de las visitas»; «Tema informático y audiovisual»; «Material adecuado a los niños».

En concreto, de las dimensiones de calidad de SERVQUAL extraídas en el grupo mixto de profesionales se han generado los siguientes atributos (Ver Fig. 1):

Fig. 1. Atributos de calidad según directivos y profesionales a partir de las dimensiones de SERVQUAL (Elaboración propia, 2015).

\begin{tabular}{|l|l|}
\hline Dimensiones SERVQUAL & \multicolumn{1}{|c|}{ Atributos de calidad } \\
\hline Aspectos tangibles & Fácil localización de la sede \\
\cline { 2 - 3 } & Adecuación de los espacios y seguridad de éstos \\
\cline { 2 - 3 } & Buen funcionamiento del equipamiento \\
\cline { 2 - 2 } & $\begin{array}{l}\text { Imagen corporativa homogénea que muestre la } \\
\text { vinculación pública }\end{array}$ \\
\cline { 2 - 3 } & Pulcritud \\
\hline Fiabilidad & Existencia del profesional de referencia \\
\cline { 2 - 3 } & Eficacia \\
\cline { 2 - 3 } & Cumplimiento de los plazos \\
\hline Capacidad de respuesta & Rapidez en las tareas administrativas \\
\cline { 2 - 3 } & Disponibilidad de los profesionales \\
\cline { 2 - 3 } & Atención profesional continuada y cercana \\
\cline { 2 - 3 } & Información suficiente a los grupos de interés. \\
\hline Seguridad & Profesionalidad \\
\cline { 2 - 3 } & Amabilidad de los profesionales \\
\cline { 2 - 3 } & Cohesión \\
\cline { 2 - 3 } & Sentido crítico \\
\hline Empatía & Confianza \\
\cline { 2 - 2 } & Atención individualizada \\
\cline { 2 - 2 } & Flexibilidad \\
\hline
\end{tabular}

Además de estos atributos, los profesionales en la sesión sin líderes sacan a relucir otros aspectos que le producen satisfacción e insatisfacción, los cuales no habían aparecido en el anterior grupo común: «Estamos bastante implicados en el trabajo porque creemos en él y en el acogimiento»; «No comparto la situación laboral»; «Genera una situación que no eliges». También se muestran preocupados por temas que no se plantean por parte de los líderes: 
«No sabemos lo que piensan». Todos muestran una actitud de conformidad en la mayoría de los puntos, concediendo una gran importancia a la motivación laboral con el trabajo desarrollado y el ámbito de actuación, excepto uno de ellos que no se siente tan desarrollado profesionalmente: «La motivación profesional y personal es grande, a pesar de la inestabilidad. Estamos insatisfechos por un lado, pero nos gusta el trabajo». En consecuencia, de esta sesión de discusión se han podido identificar dos dimensiones nuevas con sus correspondientes atributos de calidad:

- Calidad técnica: los profesionales hacen mención a la retroalimentación de su labor profesional, la formación continua y al aprendizaje de buenas prácticas.

- Calidad laboral: se incluye la motivación con el trabajo y ámbito de actuación, la estabilidad y derechos laborales, la resolución de conflictos y el liderazgo proactivo.

Durante el estudio exploratorio de la entidad, igualmente se han identificado atributos de calidad que son significativos y que convergen en gran medida con los anteriores. Dentro de los principios de calidad, todos los participantes ponen mucho énfasis en la orientación al cliente y la orientación a resultados. Los profesionales además inciden mucho en el liderazgo y en la gestión por procesos y hechos. En este último principio, aparecen algunos atributos adicionales que deben tenerse presentes: claridad en procesos y subprocesos, homogeneidad, facilidad de uso, trazabilidad y seguridad por el cumplimiento de la normativa.

En la autoevaluación, los profesionales manifiestan la dificultad del Modelo EFQM en su adaptación para el sector público y las organizaciones de voluntariado de la Junta de Andalucía (Dirección General de Administración Electrónica y Calidad de los Servicios, 2004b), así como de la herramienta de autoevaluación. En cualquier caso, la autoevaluación ha permitido confirmar y contrastar la información obtenida con anterioridad. Lo cual ha mostrado una gran coherencia en los datos obtenidos durante todo el proceso evaluativo. Los ejes prioritarios para los directivos y profesionales de de la entidad de acogimiento son: liderazgo, delimitación de objetivos y procesos, recogida sistemática de información, preocupación por la satisfacción de los grupos de interés externos y profesionales, y evaluación periódica de aspectos económicos y sobre todo, no económicos. 


\section{Discusión}

Las formas de aproximación a la calidad en la entidad evaluada han permitido conocer mejor cuál es la situación actual del servicio y comprender el contexto del acogimiento familiar. La evaluación de calidad ha permitido identificar los atributos que aportan calidad al servicio y calidad laboral a los profesionales. Además, en esta investigación ha resultado llamativo la gran similaritud en las opiniones de los directivos y los profesionales en la mayoría de los aspectos evaluados. Esto puede deberse a la gran involucración que todos presentan con los menores y a las dimensiones reducidas de la entidad, que fomenta la cercanía y el apoyo entre ambos tipos de profesionales. No obstante, los directivos muestran una percepción más positiva en cuanto al funcionamiento de la entidad. Esto evidencia que en las evaluaciones de calidad hay que complementar ambos puntos de vista para definir los atributos de calidad que deben de estar en estos servicios, siendo éstos los principales hallazgos encontrados en la evaluación.

Asimismo, se ha corroborado la idea inicial de que los modelos de calidad necesitan complementarse con indicadores propios, comunes para los Servicios Sociales Especializados. El servicio de acogimiento familiar presenta una serie de características comunes a los Servicios Sociales Especializados que deben considerarse. A saber, la orientación al cliente, la atención profesional y la coordinación externa (Ver Fig. 2).

La orientación al cliente supone en este caso poner todos los esfuerzos en procurar el bienestar de los menores, ya que no siempre las medidas de protección logran garantizar los derechos de este grupo de población (Bonal, 2010). El principio superior del bienestar del menor ha de manifestarse en los procesos que componen el servicio. La delimitación de los procesos administrativos y de sus tiempos de gestión contribuye a que el trabajo realizado sea más eficiente y satisfactorio para todos, especialmente para los menores que

Fig. 2. Características comunes de los Servicios Sociales Especializados (Elaboración propia, 2015).

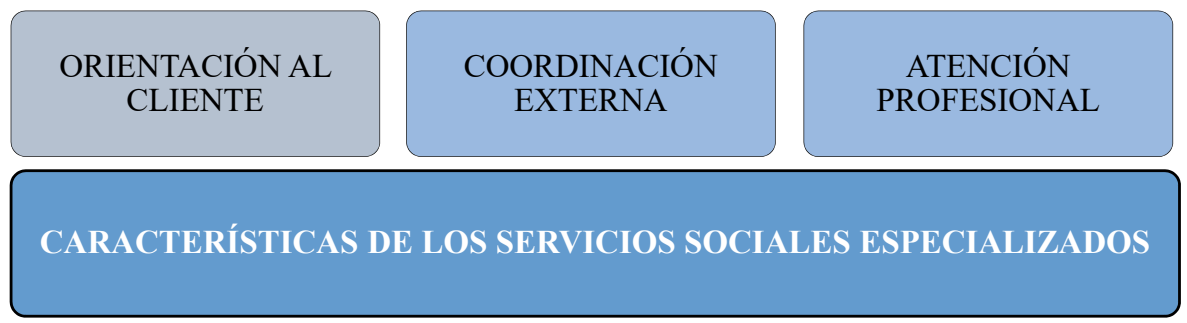

Alternativas. Cuadernos de Trabajo Social, 22, 2015, pp. 139-153 - ISSN 1133-0473 DOI: 10.14198/ALTERN2015.22.08 
han de soportar la lentitud de la burocracia, que también es la principal causa de insatisfacción para el resto de los ciudadanos según afirma la Agencia Estatal de Evaluación de las Políticas Públicas y la Calidad de los Servicios, AEVAL (2014).

En otros Servicios Sociales Especializados igualmente han de atender a un grupo de personas en una situación de vulnerabilidad social que tampoco tienen totalmente garantizados sus derechos mediante principios éticos.

En el caso de la entidad evaluada, la orientación al cliente exige además poner atención en el resto de grupos de interés: las familias acogedoras, y los propios profesionales. Sin embargo, no es muy habitual conocer la satisfacción de los profesionales del Sistema de Servicios Sociales. Pese a ello, es necesario que los profesionales se sientan contentos. En este sentido, el Parlamento Europeo (2011), señala la importancia de valorar más el trabajo de las personas que prestan servicios sociales por su gran dosis de compromiso y sensibilidad. Los profesionales participantes coinciden en que ha de reforzarse más la calidad laboral y señalan que afecta directamente en la calidad del servicio (The Social Protection Committee, 2010; Kvist, et al. 2014; Tyssen, et al., 2013). Igualmente, exponen que la motivación con el ámbito de actuación supone una de las dimensiones más valoradas en cuanto a la calidad laboral, por encima de otras como el salario, aspecto que se refleja también en otros estudios (Consejo General del Trabajo Social, 2014). También hay evidencias de que la satisfacción profesional es superior cuando la organización cuenta con un sistema de gestión de calidad como puede ser por ejemplo el Modelo Europeo (Matthies, et al., 2014).

Respecto a la atención profesional, los profesionales de Servicios Sociales Especializados como el prestado por la entidad de acogimiento evaluada, presentan unas condiciones laborales menos estables y menos recursos que si trabajasen en Servicios Sociales Comunitarios, siendo uno de los sistemas de protección más perjudicados por la crisis (Asociación Estatal de Directores y Gerentes de Servicios Sociales, 2014). De ahí a que tengan que esforzarse más si cabe en sus habilidades personales y profesionales (Pacheco Mangas y Palma García, 2014) para proporcionar una atención excelente, ya que las prácticas de intervención tradicionales impiden hacer frente a las nuevas situaciones de vulnerabilidad social (Rucabado y Heras, 2010; Sotomayor et al.,2013). Del mismo modo, en otros Servicios Sociales Especializados se requiere una atención integral centrada en la persona como señal de calidad (Martínez Fuentes, y Martínez Hernández, 2013) por parte de profesionales con las suficientes competencias técnicas y humanas, y que muestre la disponibilidad de los profesionales. Los resultados muestran que tanto los 
líderes como los profesionales dan mucha importancia a la calidad técnica de la atención prestada, en especial sobresale la dimensión de la calidad técnica que se refiere a la relación interpersonal entre quien presta el servicio y quien lo recibe (Velasco, 2011). Esta relación terapéutica hace que el profesional se adentre en los significados y comprenda a la persona que tiene delante, aspectos clave para la intervención con menores. Además este nexo de unión es un recurso en sí mismo (García Roca, 2006) que tiene una gran repercusión en la calidad del servicio (Ministerio de Sanidad, Política Social e Igualdad e IOK Laboratorio de Trabajo Social, 2012).

En este sentido, todos los participantes convergen en la conveniencia de la figura del profesional de referencia, generalmente un Trabajador Social. Esta figura goza de gran aceptación en Servicios Sociales (Brezmes, 2009; Ministerio de Sanidad, Política Social e Igualdad e IOK Laboratorio de Trabajo Social, 2012; Ministerio de Sanidad, Servicios Sociales e Igualdad, 2013), al igual que en los Servicios para la Protección de Menores. En otros estudios de Servicios Sociales, los usuarios muestran el temor a perder a este profesional y manifiestan su deseo a no tener que comenzar de nuevo (Dómenech y Giménez Bertomeu, 2012). Es decir, se valora la continuidad en la intervención.

Relacionado con lo anterior, los servicios de acogimiento familiar, al igual que el resto de Servicios Especializados, deben coordinarse con otros servicios externos. Esta situación ocasiona habitualmente problemas de coordinación, siendo necesario avanzar en establecer unos criterios homogéneos de relación y evaluación para el conjunto de Servicios Sociales Especializados, de manera que el proceso sea más provechoso para todos. En esta entidad, al depender de un servicio público, los líderes y profesionales tienen que realizar numerosas gestiones para la rendición de cuentas y para la prestación del servicio en sí, ya que los procesos realizados por la entidad son supervisados y continuados por el Servicio de Protección de Menores. Sin embargo, los problemas relacionados con la burocracia y la gestión de los tiempos, ocasionan violencia para los menores (Crespo y Moretón, 2012) que precisan de compromisos y vías de coordinación que se ajusten a la realidad.

Si centramos el análisis en las herramientas de evaluación utilizadas, la utilización de las dimensiones de SERVQUAL en los grupos de discusión, ha favorecido la aparición de bastantes atributos de calidad del servicio, así como la satisfacción de los participantes con las distintas dimensiones. Además se ha podido comprobar que las dimensiones que recoge dicha herramienta son muy apropiadas, puesto que hacen referencia a las cuestiones más significativas de cualquier servicio. Asimismo también ha resultado muy útil la aplicación más abierta de la herramienta. 
Por otro lado, la autoevaluación de la calidad sí que ha presentado una serie de inconvenientes. El modelo EFQM utilizado, pese a ser una adaptación para el sector público y las organizaciones de voluntariado, no capta todos los aspectos intrínsecos del servicio de acogimiento y es difícil de comprender. Esto coincide con la opinión de otros profesionales de Servicios Sociales Especializados que han tenido experiencias de evaluación a través del Modelo Europeo (Equipo Investigador Proyecto Gesquality, 2010). Pese a ello, la utilización de la parte de formulario de la herramienta sí que ha favorecido la valoración de las áreas de mejora y los puntos fuertes por parte de los participantes, clarificando el posterior análisis de la información. Esto coincide con otros valoraciones positivas sobre el formulario en la evaluación de la calidad (Ministerio de Administraciones Públicas, 2006).

Los resultados muestran dos puntos clave. En primer lugar, que los modelos de calidad genéricos para todo tipo de servicios presentan dificultades para ajustarse a las características de Servicios Sociales como el del acogimiento familiar. Es decir, necesitan adaptaciones para resultar más efectivos y útiles. De ahí que sea importante conocer los atributos de calidad necesarios, con el fin de avanzar en la posterior delimitación de indicadores de calidad. Relacionado con esto, en segundo lugar, la evaluación de calidad realizada ha puesto en valor las coincidencias que presentan todos los Servicios Sociales Especializados en cuanto a orientación al cliente, importancia de la calidad técnica de la atención y sobre la coordinación externa. Además ha visibilizado algunas de las condiciones laborales y la importancia de la motivación profesional en la satisfacción.

Todos estos factores nos indican que no es necesario elaborar una serie de indicadores exclusivos para el acogimiento familiar, sino que más bien conviene establecer indicadores genéricos aplicables al conjunto de servicios públicos y privados que componen los Servicios Sociales Especializados en Andalucía. Esto favorecería no sólo la comparación sino también la coordinación y evaluación de los mismos. Además, no estarían basados en las categorías habituales de atención, que no ayudan a la consolidación de los Servicios Sociales Especializados ni a la integración social de los propios usuarios. No obstante, dichos indicadores podrían complementarse con otros indicadores adicionales si se considera necesario para el servicio.

En cualquier caso, la evaluación que se ha hecho en el servicio de acogimiento familiar ha sido muy satisfactoria. El realizar la investigación en una entidad de pequeño tamaño igualmente ha servicio para evidenciar el esfuerzo que hacen los profesionales por garantizar la atención, pese a la escasez de recursos. Igualmente, la evaluación realizada ha mostrado la importancia de 
implantar la calidad en este servicio y ha favorecido el conocimiento desde las perspectivas de quienes prestan el servicio: los directivos y los profesionales. No obstante, conviene avanzar también en la evaluación de la calidad desde el punto de vista de los usuarios del servicio, ya que las nuevas necesidades sociales y las demandas de participación así lo requieren.

Las particularidades de los Servicios Sociales Especializados en cuanto a la heterogeneidad de población y formas de prestación de los servicios, hace imprescindible una mayor homogeneización en los servicios que facilite el acceso y proporcione la calidad que desde las políticas públicas se enuncia. Por este motivo, es urgente desarrollar estándares de calidad para Servicios Sociales Especializados que dignifiquen la protección social. Hagamos pues de la calidad nuestro mejor instrumento para avanzar hacia el bienestar y la justicia social de quienes más lo necesitan.

\section{Bibliografía}

AEVAL (2014). Calidad de los servicios públicos en tiempos de austeridad. Madrid: Ministerio de Hacienda y Administraciones Públicas, Agencia Estatal de Evaluación de las Políticas Públicas y la Calidad de los Servicios.

Arenas Martínez, M. (2015). Calidad percibida en los servicios sociales de inclusión. Una aproximación desde el caso de Avilés (Asturias). Cuadernos de Trabajo Social, 28-1, 113-123.

Asociación Estatal de Directores Gerentes en Servicios Sociales (2014). Índice de Desarrollo de los Servicios Sociales 2014. Asociación Estatal de Directores Gerentes en Servicios Sociales.

BARRANCO EXPÓSITO C. (2011). Buenas prácticas de calidad y Trabajo Social. Alternativas. Cuadernos de Trabajo Social, 18, 57-74.

BREZMES Nieto M.J. (2009). Propuesta para la configuración de un modelo actualizado de Servicios Sociales Municipales. Zamora: Federación Española de Municipios y Provincias.

Consejo General de Trabajo Social (2014) I Informe sobre los Servicios Sociales en España. Madrid: Consejo General de Trabajo Social.

Crespo Garrido, M., Moretón SAnZ, F. (2012). La nueva Ley de Servicios Sociales en Castilla La Mancha: la figura del Trabajador Social como protección de la calidad de vida del menor. Revista de Estudios de Juventud, 97, 207-222.

Cruz Roja Española (2010). Manual de buena práctica en acogimiento familiar. Madrid: Cruz Roja Española.

Del VAlLE, J.F., BRAVO, A., y LÓPEZ, M. (2009). El acogimiento familiar en España: implantación y retos actuales. Papeles del Psicólogo, 30-1, 33-41.

Dirección General de Administración Electrónica y Calidad de los Servicios (2004a). 
Cuestionario de Autoevaluación. Sevilla: Consejería de Justicia y Administración Pública.

Dirección General de Administración Electrónica y Calidad de los Servicios (2004b). Modelo EFQM de Excelencia en la Junta de Andalucía. Sevilla: Consejería de Justicia y Administración Pública.

Dómenech López, Y., Giménez Bertomeu, V. (2012). Percepciones sobre la calidad de los Servicios Sociales de las personas jóvenes usuarias: utilidades para el diseño de estándares de calidad. Revista de Estudios de Juventud, 97, 123-146.

Equipo Investigador Proyecto GESQUALITY (2010). Aplicación del modelo europeo EFQM de excelencia en centros residenciales y en el servicio de ayuda a domicilio: Diseño de procesos y cuadro de mando integral. Memoria de investigación. Barcelona: Fundación SAR.

European Commission (2013). Social Policies. Social Europe guide. Volume 5. Belgium: European Commission.

Fundación Edad y Vida (2008). Calidad y acreditación para las entidades prestadoras de servicios de atención a las personas mayores en situación de dependencia. Madrid: IMSERSO.

GARCÍA RoCA, J. (2006). Memorias silenciadas en la construcción de los servicios sociales. Cuadernos de Trabajo Social, 19, 197-212.

Kvist, T., Voutilainen, A., MÄntynen, R. y VeHViläInEn-Julkunen, K. (2014). The relationship between patients' perceptions of care quality and three factors: nursing staff job satisfaction, organizational characteristics and patient age. BMC Health Services Research 14, 466.

Martínez Fuentes, M. T., Martínez Hernández, L. (2013). Promoción del desarrollo infantil y atención temprana: calidad de los servicios. Azarbe. Revista Internacional de Trabajo Social y Bienestar, 2, 49-67.

Matthies Baraibar, C., Arcelay Salazar, A., Cantero González, D., Colina Alonso, A., García Urbaneja, M., GonzÁlez llinares, M., et. al. (2014). Is organizational progress in the EFQM model related to employee satisfaction?. BMC Health Services Research, 14, 468.

Medina Tornero, M. E. (2006). La implantación de la calidad en Servicios Sociales. Desafío a la tradición. En Gobierno de la Rioja (Ed.), III Jornadas sobre la Calidad en Centros de Servicios Sociales (pp.121-137). Logroño: Gobierno de La Rioja.

Ministerio de Administraciones Públicas (2006). Guía de orientación para la realización de estudios de análisis de la demanda y de encuestas de satisfacción. Madrid: Ministerio de Administraciones Públicas.

Ministerio de Sanidad, Política Social e Igualdad, IOK Laboratorio de Trabajo Social (2012). Calidad en los Servicios Sociales de Atención Primaria. Madrid: Ministerio de Sanidad, Política Social e Igualdad. 
Ministerio de Sanidad, Servicios Sociales e Igualdad (2013). Catálogo de referencia de Servicios Sociales. Madrid: Ministerio de Sanidad, Servicios Sociales e Igualdad.

Miranda González, F., Chamorro Mera, A. y Rubio lacoba, S. (2007). Introducción a la gestión de la calidad. Madrid: Delta Publicaciones Universitarias.

Pacheco Mangas, J. y Palma García, M. (2014). El discurso profesional en Servicios Sociales Comunitarios: una aproximación desde la identificación de repertorios interpretativos. Alternativas. Cuadernos de Trabajo Social, 21, 9-28.

Parlamento Europeo (2011). Informe sobre el futuro de los servicios sociales de interés general (Informe no. 2009/2222 INI). Bruselas: Parlamento Europeo.

Rucabado Sala, A., Heras Robles, I. (2010). La atención a los grupos vulnerables en Jaén. Jaén: Universidad de Jaén.

Sotomayor Morales, E., De la Fuente Robles, Y., García Domingo, M., Grande Gascón, M. y Alberich Nistal, T. (2013). Calidad en los servicios de bienestar en un contexto de crisis económica internacional. Comunitania. Revista internacional de Trabajo Social y Ciencias Sociales, 5, 153-179.

The Social Protection Comitee (2010). A Voluntary European Quality Framework for Social Services. (Report SPC/2010/10/8 final, 8 October). Luxemburg: The Social Protection Comitee.

Tyssen, R., Palmer, K., Solberg, I., Voltmer, E. y Frank, E. (2013). Physicians' perceptions of quality of care, professional autonomy, and job satisfaction in Canada, Norway, and the United States. BMC Health Services Research, 13, 516.

Velasco SÁnCHEZ, J. (2011). Gestión de la calidad. Mejora continua y sistemas de gestión. Teoría y práctica. Madrid: Ediciones Pirámide.

Zeithaml, V., Parasuraman, A. y Berry, L. (1992). Calidad Total en la Gestión de Servicios. Madrid: Díaz de Santos S. A. 